\section{Commentary: That's going to leave a mark, but how big?}

\author{
Kathryn E. Engelhardt, MD, MS, and \\ Bryan Meyers, MD
}

Targeted immunotherapy is changing the prognosis of lung cancer. Tyrosine kinase inhibitors (TKIs) like osimertinib can offer dramatic tumor control for patients with epidermal growth factor reception mutations. ${ }^{1}$ Current treatment guidelines do not support TKIs in the neoadjuvant setting, but this strategy may be considered after multidisciplinary discussion. As data emerge from "off-protocol" use, guidelines may change if the results prove promising. From a surgical perspective, we consider both the effect of neoadjuvant therapy on oncologic outcomes and the short-term tissue fibrosis that may hinder the surgery or increase complications. Improved oncologic downstaging may be less desirable if neoadjuvant TKI therapy renders too many surgically unresectable by inflammation and fibrosis.

In this JTCVS Techniques, Chudgar and Jones share 2 cases treated with neoadjuvant osimertinib followed by surgery for non-small cell lung cancer with epidermal growth factor reception mutation or deletion. ${ }^{2}$ They encountered varied responses and a seemingly unpredictable effect regarding the technical difficulty of the resection. One patient had only 3 months of therapy and had significant fibrosis with loss of tissue planes, whereas the other had 7 months of therapy with little impact on the technical difficulty at resection. Interestingly, the patient with 3 months of therapy had much less apparent tumor effect than the patient with 7 months of therapy, who had regression in primary tumor site, loss of positron emission tomography

From the Division of Cardiothoracic Surgery, Department of Surgery, Washington University, Washington University School of Medicine/Barnes-Jewish Hospital, Saint Louis, Mo.

Disclosures: The authors reported no conflicts of interest.

The Journal policy requires editors and reviewers to disclose conflicts of interest and to decline handling or reviewing manuscripts for which they may have a conflict of interest. The editors and reviewers of this article have no conflicts of interest.

Received for publication Feb 10, 2021; revisions received Feb 10, 2021; accepted for publication Feb 22, 2021; available ahead of print Feb 27, 2021.

Address for reprints: Bryan Meyers, MD, Division of Cardiothoracic Surgery, Department of Surgery, Washington University, Washington University School of Medicine/Barnes-Jewish Hospital, Campus Box 8234, 660 South Euclid Ave, Saint Louis, MO 63110-1093 (E-mail: meyersb@wustl.edu).

JTCVS Techniques 2021;7:299-300

2666-2507

Copyright (C) 2021 The Authors. Published by Elsevier Inc. on behalf of The American Association for Thoracic Surgery. This is an open access article under the CC BY-NCND license (http://creativecommons.org/licenses/by-nc-nd/4.0/).

https://doi.org/10.1016/j.xjtc.2021.02.043

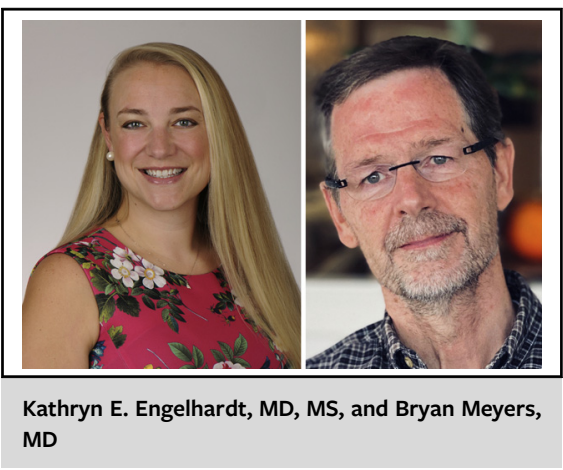

CENTRAL MESSAGE

Tyrosine kinase inhibitors may be used in the neoadjuvant setting as data become available. The effect of TKIs on fibrosis and tissue planes may be unpredictable, as this small series shows.

avidity, and absence of cancer in the previously positive lymph nodes.

The authors suggest that these observations indicate that the effect of osimertinib on fibrosis is unpredictable. It is important to also consider other possible explanations for these findings. The patient with the 3-month course had biopsy-proven positive lymph nodes and a limited response to his neoadjuvant treatment, having been downstaged trivially from cT2aN2M0 to ypT1bN2M0 with $60 \%$ viable tumor on pathology. This patient was noted to have extensive fibrotic changes and loss of tissue planes requiring sharp dissection. In contrast, the patient treated for 7 months had clinically positive nodes based on positron emission tomography/computed tomography but preoperative biopsies were negative for cancer. Of course, this could be a sampling error, but it could also have been an inflammatory lymph node rather than a metastatic lymph node. This patient was effectively downstaged from cT2bN1 to ypT1aN0 with $10 \%$ viable tumor on pathology. At surgery, this patient was noted to have preserved tissue planes, less tedious of a nodal dissection, shorter operative time, and lower blood loss. The differences could be due to the biology of each patient's tumor rather than the result of the treatment effect for that tumor. The challenging dissection could have been due to an increased tumor burden in the lymph nodes of the 3 month patient. It is hard to draw conclusions from 2 patients. 
TKIs are likely to be used more frequently in the neoadjuvant setting; it will be important to track perioperative findings and outcomes to understand better how these medications will affect our patients. This manuscript is an excellent start.

\section{References}

1. Soria J-C, Ohe Y, Vansteenkiste J, Soria J-C, Ohe Y, Vansteenkiste J, et al. Osimertinib in untreated EGFR-mutated advanced non-small-cell lung cancer. $N$ Engl J Med. 2017;378:113-25.

2. Chudgar NP, Jones DR. Thoracoscopic lobectomy following neoadjuvant tyrosine kinase inhibitor treatment. J Thorac Cardiovasc Surg Tech. 2021;7:294-7. 